\title{
Effectiveness and safety of catheter removal alone versus standard anticoagulation therapy after catheter removal for peripherally inserted central catheter (PICC)-related thrombosis
}

\author{
Li Liu ${ }^{1}$, Jing Huang ${ }^{1}$, Zhoupeng $\mathrm{Wu}^{2}$, Yukui $\mathrm{Ma}^{2}$ \\ ${ }^{1}$ Department of Vascular Surgery, West China Hospital, Sichuan University/West China School of Nursing, Sichuan University, Chengdu, China; \\ ${ }^{2}$ Department of Vascular Surgery, West China Hospital, Chengdu, China \\ Contributions: (I) Conception and design: Z Wu; (II) Administrative support: Y Ma; (III) Provision of study materials or patients: Z Wu, L Liu; (IV) \\ Collection and assembly of data: L Liu, J Huang; (V) Data analysis and interpretation: L Liu, Z Wu; (VI) Manuscript writing: All authors; (VII) Final \\ approval of manuscript: All authors. \\ Correspondence to: Zhoupeng Wu, MD. Department of Vascular Surgery, West China Hospital, 37 Guoxue Alley, Chengdu 610041, China. \\ Email: lzwzp19@163.com.
}

Background: Currently, peripherally inserted central catheters (PICCs) are widely used; however, there are associated problems due to catheter-related thrombosis (CRT). According to the existing literature and guidelines, 3-6 months of anticoagulation therapy is recommended, but these recommendations are based on analogous deep vein thrombosis of the lower limbs. More specific management strategies need to be developed, and the safety and effectiveness of these strategies needs to be investigated.

Methods: Some studies have suggested that catheter removal alone is a reasonable option, especially for patients with a higher risk of bleeding. We conducted a retrospective study of hospitalized patients from a single center who were diagnosed with PICC-related thrombosis. Among the 85 patients who met the inclusion criteria, 63 patients were treated with catheter removal alone, and 22 patients received anticoagulation therapy after catheter removal. The progression of thrombosis and bleeding in the two groups were compared. Most patients who underwent catheter removal alone had hematological malignancies, and thrombocytopenia had occurred during chemotherapy.

Results: After PICC removal, no patients in the anticoagulation therapy group developed progressive thrombosis, while 10 patients in the catheter removal alone group developed progressive thrombosis or pulmonary embolism (PE), including one case of $\mathrm{PE}$, four cases of secondary upper extremity deep vein thrombosis (UEDVT), and five patients showed obvious aggravation of thrombosis after catheter removal, and were administered repeated anticoagulant therapy. In the PICC removal + anticoagulation treatment group, major bleeding increased significantly (28.6\% vs. $4.7 \%, \mathrm{P}=0.006)$.

Conclusions: Compared to the PICC removal + anticoagulation treatment group, the risk of major bleeding in the catheter removal alone group was significantly reduced. In some patients with an increased bleeding risk, catheter removal alone may be a safer and more effective option than catheter removal with anticoagulation treatment for CRT.

Keywords: Peripherally inserted central catheter (PICC); anticoagulation; thrombosis

Submitted Oct 28, 2021. Accepted for publication Dec 10, 2021.

doi: 10.21037/atm-21-5884

View this article at: https://dx.doi.org/10.21037/atm-21-5884 


\section{Introduction}

Patients who had implantation of peripherally inserted central catheter (PICC) are susceptible to catheterrelated thrombosis (CRT), for they usually had two major risk of thrombosis, that is, tumor and venous catheter. The current guidelines from three different professional associations recommend that patients with CRT should be treated with anticoagulation for 3-6 months after catheter removal (1-3). However, these recommendations are inferred from various studies of deep vein thrombosis of the lower extremities, and there is currently a lack of prospective research data on the treatment of CRT. There are differences between CRT and deep vein thrombosis of the lower extremities, suggesting that different management methods for the two types of thrombosis may be reasonable. Firstly, CRT is often missed. In patients with undiagnosed CRT, CRT is often unintentionally discovered only by catheter removal, and no obvious clinical symptoms are identified. For example, a prospective study of patients with tumors with PICC found that $51.4 \%$ of the patients had CRT during an ultrasound examination, and $45.6 \%$ of the patients were asymptomatic (4). Secondly, compared with catheter removal alone, coagulation may not change the development of CRT. A study involving follow-up ultrasound scans for CRT showed that anticoagulation therapy did not increase the rate of thrombus regression compared with simple catheter removal $(5,6)$. Thirdly, CRT is associated with significantly less risk of pulmonary embolism (PE) than deep vein thrombosis of the lower extremities (3\% and $16 \%$ in two studies); therefore, any anticoagulant treatment to reduce this potential risk is not very effective (7). Fourthly, comorbidities in patients with PICCs are often complicated; thus, bleeding is more likely to occur. A recent meta-analysis of multiple studies involving patients with CRT treated with anticoagulation therapy found that the incidence of major bleeding was as high as $4.9 \%$ (8). Other reports have also demonstrated that the rate of major bleeding may be close to $25 \%$ (5).

At present, CRT is very common, and is usually only unintentionally discovered during catheter removal, without clinical symptoms. Ultrasound is usually needed to detect CRT. Therefore, the risk of CRT is very small, and anticoagulation therapy has associated bleeding risks. Therefore, many medical staff use catheter removal alone to treat PICC-related thrombosis. The effectiveness and safety of catheter removal alone versus continued anticoagulation therapy after catheter removal remains unknown. To better understand the risks and benefits of these two strategies, we conducted a retrospective study of inpatients with PICCrelated thrombosis. A large number of patients in China need PICC implantation every year, but there has been a lack of relevant domestic research on the anticoagulation problem after PICC extubation. Although this study is a retrospective study, it also has certain reference value for domestic problems. We present the following article in accordance with the STROBE reporting checklist (available at https://dx.doi.org/10.21037/atm-21-5884).

\section{Methods}

All procedures performed in this study involving human participants were in accordance with the Declaration of Helsinki (as revised in 2013). This study was approved by the Ethics Institutional Review Board of the West China Hospital of Sichuan University (approval No. 000053291). All patients provided consent to be included in the study, and also to have their data published. All patients signed the statement of consent form. We conducted a retrospective review of patients diagnosed with CRT from June 2018 to April 2020. Patients diagnosed with CRT were identified through case database records, which were then reviewed and confirmed. We only included patients older 18 years who had PICC-related thrombosis confirmed by ultrasound during hospitalization. We excluded patients with catheters inserted through a central vein, patients diagnosed in outpatient clinics, as well as patients with progressive venous thromboembolism (VTE) at the time of CRT diagnosis, trauma, and known thrombotic-related accidents [such as heparin-induced thrombocytopenia (HIT), and obvious bleeding]. Patients who required vascular surgery and thrombolytic therapy during treatment also were excluded. In addition to recording routine basic data, a complete evaluation of the medical records was performed to determine the incidence of secondary VTE after PICC removal and the incidence of severe bleeding, as defined by the International Thrombosis and Hemostasis Association (9). The diagnosis of CRT was established by duplex ultrasound. The outcome of major bleeding event was checked by telephone or out-patient follow-up. Progress of thrombosis was evaluated by duplex ultrasound.

\section{Statistical analysis}

Frequencies and percentages were used to represent univariate categorical variables, and means and standard 


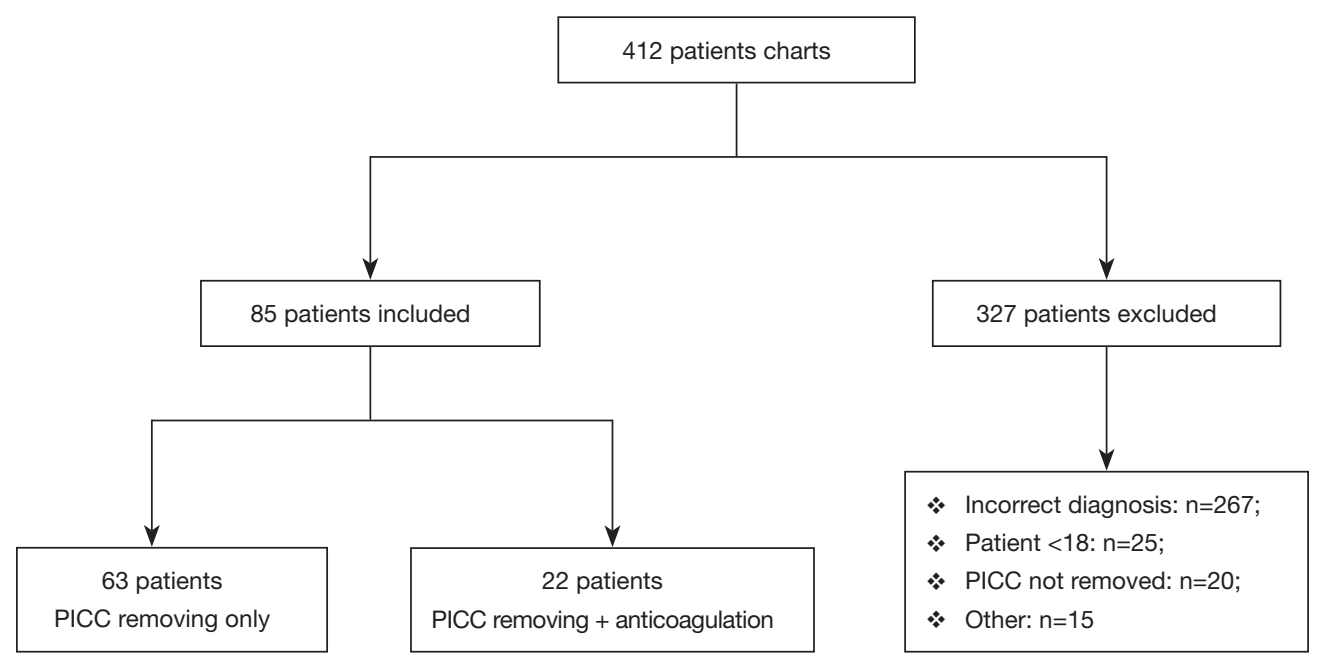

Figure 1 Inclusion/exclusion criteria. A total of 412 charts were reviewed for potential inclusion, of which 327 were excluded for various reasons. PICC, peripherally inserted central catheter.

deviations were used to represent interval variables. In addition, an independent Student's $t$-test was used to test the differences between interval variables. When it was assumed that the ordinal categories in the variables represented a basic normal distribution, Pearson productmoment correlation and multiple regression analysis were used to analyze the data. Moreover, the chi-squared test and Kendall's rank test were used for ordinal variables. Finally, all results were analyzed using SPSS software (version 18.0). It should be noted that a $P$ value of less than 0.05 was considered statistically significant.

\section{Results}

After consulting the medical records of 412 patients, 85 patients were ultimately included. The most common reasons for exclusion included non-PICC-related thrombosis (commonly a coding error), patients younger than 18 years, and patients receiving thrombolytic therapy (Figure 1). Of the included patients, 63 (74.1\%) underwent PICC removal alone, while 22 (25.9\%) underwent PICC removal plus anticoagulation therapy. Table 1 summarizes the basic information for the diagnosis of CRT. Patients who underwent PICC removal alone were more likely to have hematological malignancies, have received chemotherapy at the time of admission, and have platelet counts $<50 \times 10^{9} / \mathrm{L}$ at the time of admission, and were less likely to have proximal thrombosis.

The efficacy and safety results of the two comparative studies are listed in Table 2. In total, $15.9 \%$ of patients who underwent catheter removal alone developed progressive VTE; one patient developed PE (1.6\%), four developed secondary upper extremity deep vein thrombosis (UEDVT) (6.3\%), and five developed VTE. Thrombus progression required repeated anticoagulant therapy in $7.9 \%$ of patients. The symptoms of one patient in the anticoagulation group $(4.5 \%)$ progressed, leading to the need for repeated clinical evaluation after removal. Seven patients $(31.8 \%)$ in the anticoagulation group and three patients $(4.8 \%)$ in the catheter removal alone group had major bleeding $(\mathrm{P}=0.006)$. No patients died of thrombosis or bleeding.

\section{Discussion}

In a retrospective study of hospitalized patients with CRT, PICC removal alone was associated with more secondary VTE events and worse symptoms than PICC removal plus anticoagulation therapy. The incidence of repeated anticoagulation did not increase significantly. However, in patients who underwent PICC removal plus anticoagulation therapy, we observed a markedly higher incidence of severe bleeding, indicating that there is a significant risk of bleeding in some populations using this strategy. This study indicates that for some patients, especially those with an increased risk of bleeding, PICC removal alone may be a viable treatment option. Treatment for CRT involves the following three factors: significantly improving patient symptoms, preventing progressive VTE, and reducing the 
Table 1 Characteristics of the 85 included patients

\begin{tabular}{|c|c|c|}
\hline Characteristics & $\begin{array}{l}\text { PICC removal + anticoagulation therapy } \\
\text { (number/\% or mean) }\end{array}$ & $\begin{array}{l}\text { PICC removal only } \\
\text { (number/\% or mean) }\end{array}$ \\
\hline Total & 22 & 63 \\
\hline \multicolumn{3}{|l|}{ Sex } \\
\hline Mean age (years) & 54.5 & 58.7 \\
\hline \multicolumn{3}{|l|}{ Comorbidities } \\
\hline Prior history of VTE & $2 / 9.1$ & $3 / 4.8$ \\
\hline Obesity & $9 / 40.9$ & $14 / 22.2$ \\
\hline Surgery while admitted & $5 / 22.7$ & $10 / 15.9$ \\
\hline Estrogen before admission & $2 / 9.1$ & $2 / 3.2$ \\
\hline \multicolumn{3}{|l|}{ Malignancy } \\
\hline Leukemia & $4 / 18.2$ & $28 / 44.4$ \\
\hline Lymphoma & 2/9.1 & $8 / 12.7$ \\
\hline Myeloma & $0 / 0$ & $9 / 14.3$ \\
\hline Prophylactic & $8 / 36.4$ & $17 / 27.0$ \\
\hline Platelet count (mean) & 246 & 133 \\
\hline \multicolumn{3}{|l|}{ Thrombosis location } \\
\hline Internal jugular & $5 / 22.7$ & $4 / 6.3$ \\
\hline Subclavian & $11 / 50.0$ & $23 / 36.5$ \\
\hline Axillary & $3 / 13.6$ & $19 / 30.2$ \\
\hline Brachial & $3 / 13.6$ & $17 / 27.0$ \\
\hline \multicolumn{3}{|l|}{ Treatment } \\
\hline Enoxaparin & $9 / 40.9$ & - \\
\hline Warfarin & $3 / 13.6$ & - \\
\hline DOAC & $10 / 45.5$ & - \\
\hline
\end{tabular}

PICC, peripherally inserted central catheter; VTE, venous thromboembolism; PAD, peripheral artery disease; DOAC, direct oral anticoagulant. 
Table 2 Efficacy and safety of the two treatment methods in the 85 included patients

\begin{tabular}{|c|c|c|c|}
\hline The adverse events & $\begin{array}{l}\text { PICC removal + anticoagulation } \\
\text { therapy (number/\%) }\end{array}$ & $\begin{array}{l}\text { PICC removal only } \\
\text { (number/\%) }\end{array}$ & $P$ \\
\hline Total & 22 & 63 & \\
\hline \multicolumn{4}{|l|}{ Progressive thrombosis } \\
\hline Total & $1 / 4.5$ & $10 / 15.9$ & 0.45 \\
\hline $\begin{array}{l}\text { Obvious aggravation of } \\
\text { thrombosis }\end{array}$ & $1 / 4.5$ & $5 / 7.9$ & 1.08 \\
\hline Bleeding & & & 0.006 \\
\hline Other & $2 / 9.1$ & $2 / 3.2$ & \\
\hline
\end{tabular}

PICC, peripherally inserted central catheter; DVT, deep vein thrombosis.

risk of bleeding (10).

The treatment of symptoms associated with CRT is an important aspect. The majority of patients who underwent PICC removal alone showed significant relief of their symptoms after PICC removal, and only a small number of patients experienced aggravation of their symptoms and needed to restart anticoagulation therapy. Due to the limitations of this retrospective review, we could not determine the proportion of patients diagnosed with CRT with symptoms; however, if symptoms are the basis for preliminary screening for CRT, this may be the vast majority. Therefore, for most symptomatic CRT patients, simple PICC removal seems to be a reasonable strategy, but it should be understood that a small number of patients will have progressively worsening symptoms and need to restart anticoagulation therapy. There are very few studies on post-thrombotic syndrome caused by CRT, and the range of related morbidity reported in the literature has also changed greatly (11). Our current study showed that it is not common for patients who undergo PICC removal alone to continue to have obvious symptoms. Furthermore, our study excluded patients who received catheter thrombolysis because these patients are more likely to have corresponding symptoms.

A second consideration for the treatment of CRT is the prevention of secondary or progressive VTE events. Although we observed a small incidence of secondary VTE events in the catheter removal alone group, no fatal VTE events were observed. Among the hospitalized patients in our study, based on their baseline data, their risk of VTE may be very high (12). The current guidelines recommend that preventive doses of anticoagulants be routinely used for most patients during hospitalization because the literature has shown their effectiveness for VTE prevention (13). If the risk of bleeding is not high, the use of this drug can be resumed after catheter removal. This strategy seems reasonable.

Finally, bleeding is the main problem with the use of anticoagulant drugs. Our research included patients with complex underlying diseases, many of whom had cancer, received chemotherapy on admission to the hospital, and had thrombocytopenia. In such patients, the use of anticoagulant drugs is challenging. There is no clear consensus or guidelines on how to adjust for thrombocytopenia caused by chemotherapy drugs or how to adjust anticoagulation strategies and use platelet transfusions (14). The bleeding rate in this study was significantly higher than that in previous studies involving cancer patients with CRT $(15,16)$. This variability may be attributed to the main difference in the patient population in this study; the studies in the abovementioned literature were conducted in outpatient settings, excluded patients with severe thrombocytopenia, and included patients with a lower risk of bleeding. In the general population, other 
retrospective reports on CRT have also reported a similar bleeding rate of close to $25 \%$. In view of the above research results and the existing literature, and considering the bleeding risk associated with anticoagulation, PICC removal alone may be a reasonable choice for patients with an increased risk of bleeding. To sum up, our results suggested that catheter-removal alone was associated with significantly decreased risk of major bleeding compared with catheterremoval plus anticoagulation. Therefore, we recommended catheter removal alone may be an option for CRT in select patients with high risk of bleeding.

\section{Conclusions}

A limitation of this study is that it was a retrospective study with a small sample size. Also, there was a significant difference between the PICC removal alone and the PICC removal + anticoagulation therapy cohorts, indicating that doctors may have been worried that due to the emergence of thrombocytopenia, the risk of adverse consequences related to active treatment could have been higher. Furthermore, this study showed that for some hospitalized patients with PICC-related thrombosis, PICC removal may be a viable option. More prospective studies should be conducted to evaluate the effectiveness and safety of PICC removal alone.

\section{Acknowledgments}

Funding: None.

\section{Footnote}

Reporting Checklist: The authors have completed the STROBE reporting checklist. Available at https://dx.doi. org/10.21037/atm-21-5884

Data Sharing Statement: Available at https://dx.doi. org/10.21037/atm-21-5884

Conflicts of Interest: All authors have completed the ICMJE uniform disclosure form (available at https://dx.doi. org/10.21037/atm-21-5884). The authors have no conflicts of interest to declare.

Ethical Statement: The authors are accountable for all aspects of the work in ensuring that questions related to the accuracy or integrity of any part of the work are appropriately investigated and resolved. All procedures performed in this study involving human participants were in accordance with the Declaration of Helsinki (as revised in 2013). This study was approved by the Ethics Institutional Review Board of the West China Hospital of Sichuan University (approval No. 000053291). All patients provided consent to be included in the study, and also to have their data published. All patients signed the statement of consent form.

Open Access Statement: This is an Open Access article distributed in accordance with the Creative Commons Attribution-NonCommercial-NoDerivs 4.0 International License (CC BY-NC-ND 4.0), which permits the noncommercial replication and distribution of the article with the strict proviso that no changes or edits are made and the original work is properly cited (including links to both the formal publication through the relevant DOI and the license). See: https://creativecommons.org/licenses/by-nc-nd/4.0/.

\section{References}

1. Sousa B, Furlanetto J, Hutka M, et al. Central venous access in oncology: ESMO Clinical Practice Guidelines. Ann Oncol 2015;26 Suppl 5:v152-68.

2. Schiffer CA, Mangu PB, Wade JC, et al. Central venous catheter care for the patient with cancer: American Society of Clinical Oncology clinical practice guideline. J Clin Oncol 2013;31:1357-70.

3. Kearon C, Akl EA, Ornelas J, et al. Antithrombotic Therapy for VTE Disease: CHEST Guideline and Expert Panel Report. Chest 2016;149:315-52.

4. Liu Y, Gao Y, Wei L, et al. Peripherally inserted central catheter thrombosis incidence and risk factors in cancer patients: a double-center prospective investigation. Ther Clin Risk Manag 2015;11:153-60.

5. Jones MA, Lee DY, Segall JA, et al. Characterizing resolution of catheter-associated upper extremity deep venous thrombosis. J Vasc Surg 2010;51:108-13.

6. Malinoski DJ, Ewing T, Patel MS, et al. The natural history of upper extremity deep venous thromboses in critically ill surgical and trauma patients: what is the role of anticoagulation? J Trauma 2011;71:316-21; discussion 321-2.

7. Joffe HV, Kucher N, Tapson VF, et al. Upper-extremity deep vein thrombosis: a prospective registry of 592 patients. Circulation 2004;110:1605-11.

8. Kahale LA, Tsolakian IG, Hakoum MB, et al. 
Anticoagulation for people with cancer and central venous catheters. Cochrane Database Syst Rev 2018;6:CD006468.

9. Schulman S, Kearon C; Subcommittee on Control of Anticoagulation of the Scientific and Standardization Committee of the International Society on Thrombosis and Haemostasis. Definition of major bleeding in clinical investigations of antihemostatic medicinal products in non-surgical patients. J Thromb Haemost 2005;3:692-4.

10. Bauersachs R, Schellong SM, Haas S, et al. CERTIFY: prophylaxis of venous thromboembolism in patients with severe renal insufficiency. Thromb Haemost 2011;105:981-8.

11. Heit JA, Melton LJ 3rd, Lohse CM, et al. Incidence of venous thromboembolism in hospitalized patients vs community residents. Mayo Clin Proc 2001;76:1102-10.

12. Elman EE, Kahn SR. The post-thrombotic syndrome after upper extremity deep venous thrombosis in adults: a systematic review. Thromb Res 2006;117:609-14.

13. Kahn SR, Lim W, Dunn AS, et al. Prevention of VTE

Cite this article as: Liu L, Huang J, Wu Z, Ma Y. Effectiveness and safety of catheter removal alone versus standard anticoagulation therapy after catheter removal for peripherally inserted central catheter (PICC)-related thrombosis. Ann Transl Med 2021;9(24):1778. doi: 10.21037/atm-21-5884 in nonsurgical patients: Antithrombotic Therapy and Prevention of Thrombosis, 9th ed: American College of Chest Physicians Evidence-Based Clinical Practice Guidelines. Chest 2012;141:e195S-226S.

14. Li A, Davis C, Wu Q, et al. Management of venous thromboembolism during thrombocytopenia after autologous hematopoietic cell transplantation. Blood Adv 2017;1:707-14.

15. Davies GA, Lazo-Langner A, Gandara E, et al. A prospective study of Rivaroxaban for central venous catheter associated upper extremity deep vein thrombosis in cancer patients (Catheter 2). Thromb Res 2018;162:88-92.

16. Kovacs MJ, Kahn SR, Rodger M, et al. A pilot study of central venous catheter survival in cancer patients using low-molecular-weight heparin (dalteparin) and warfarin without catheter removal for the treatment of upper extremity deep vein thrombosis (The Catheter Study). J Thromb Haemost 2007;5:1650-3. 